Council's organization and methods which lasted nearly two years. Only in the clerical and typing grades were any posts considered redundant by the investigators.

Besides the withdrawal from Czechoslovakia and Bulgaria, the feature of the Council's work in Europe during 1950-51 was the opening of a new branch in Düsseldorf to take over the work previously done by the cultural department of the High Commission in Germany. The European work bore a major share of the reduction made in the Council's grant-in-aid as a whole, and the European budget for 1950-51 was about $£ 100,000$ less than that for the previous year. With Latin America this budget still accounts for more than half the Council's expenditure on overseas services, though expenditure in the Middle East comes next to that in Europe in magnitude. Lack of funds compelled withdrawal from the Sudan. Outside education in its narrower sense, the report suggests that the bringing of specialists and influential visitors to Great Britain and the giving of lectures, with supporting illustrative material, on agriculture and its associated sciences are the best means of maintaining and strengthening the interest of the Middle Eastern countries in the culture and institutions of the United Kingdom. In the Far East, with the exception of China, where a marked increase in the work of the local centres at Nanking, Shanghai and Peking was noted during the year in spite of Government interference with the Council's work, the main difficulty has been lack of funds; but as regards Latin America, commenting on the value of British publications for the propagation of knowledge, the report observes that in some countries currency restrictions or complicated import requirements constitute the chief obstacle and one which the Council can do little to overcome.

The British Council is responsible for the operation of schemes for the interchange of university teachers, scholars and scientific workers under the cultural conventions into which Great Britain has entered. Thirty-nine regular interchange visits of this type are already arranged each year with Austria, Belgium, France, Italy, the Netherlands and Norway. Negotiations are proceeding with Denmark, Western Germany, Spain, Sweden and Switzerland, which would bring the total to fifty-two, while under a scheme established at the request of the Conference of the Universities of the British Commonwealth at Oxford in 1948, thirty-three university teachers from oighteen Commonwealth universities visited the United Kingdom and one scholar from Britain visited Commonwealth universities. Seventy-two scholarships were offered to British students by Austria, Belgium, Brazil, Denmark, Finland, France, Germany, Italy, the Netherlands, Norway, Persia, Portugal, Spain, Sweden and Switzerland, in return for the scholarships offered by the Couneil for many years to students from foreign universities. The number of advisory visits has also increased, and the report cites in illustration those to France on recent developments in stock-breeding ; and visits of a team of three surgeons, led by Mr. C. Price Thomas, to Yugoslavia; of Dame Katharine Watt to India, Pakistan, Syria, Egypt, Iraq, Jordan and the Lebanon, to advise on nursing services; and of Dr. Mouat Jones to the Middle Fast and India, to advise on the development of technical education.

The net expenditure of the Council during 1949-50, the last year for which figures of actual expenditure are available, was $£ 3,045,321$, and the total sum available from public funds for 1950-51 was $£ 3,233,700$. There were, however, considerable changes in the distribution of expenditure, the estimates providing for major expansions in the work carried out for the Colonial Office, the Commonwealth Relations Office and Colonial Development and Welfare, and for a contraction, under instructions, of the provision for work in foreign countries of $£ 325,000$; the devaluation of sterling added a further $£ 206,000$ to this last figure. In the estimates for 1951-52, the Council has been called upon to reduce the provision for work in foreign countries by a further $£ 364,000$, although the Foreign Office is pressing for the expenditure of $£ 49,000$ on new services regarded as of the highest importance. Such reductions must entail drastic changes, and it is a matter for regret that neither in this report nor elsewhere is there any indication of a real inquiry into how far there is overlapping in the work of the British Council with that of the Central Office of Information, the Foreign Office itself, and the Overseas Service of the British Broadcasting Corporation which could be eliminated to the general advantage.

\section{ELECTRONICS IN NUCLEAR SCIENCE AND ENGINEERING}

$\mathrm{M}$

ANY visitors from overseas have been attracted to Great Britain in this Festival Year, not only to attend the Festival, but also to participate in the various supporting conferences arranged by the professional, scientific and technical institutes. The British Institution of Radio Engineers, the silver jubilee of which was to have been held last year, delayed its celebrations of this event and planned instead a Radio Convention for 1951. The Council of the Institution considered it appropriate that the Convention should aim at covering the whole field of electronics. In fact, the conference was probably the most comprehensive in its field ever to be held in Great Britain, and more than seventy papers were presented covering applications of electronics in all fields from nuclear physics to audio-frequency engineering. All the papers are being published in the Journal of the Institution.

The first session of the Convention was held at University College, London, during July 3 and 4 and dealt with the applications of electronics in nuclear science and engineering. The chair at these meetings was taken by Dr. Denis Taylor, of the Atomic Energy Research Establishment, Harwell, and his address, which was illustrated by lantern slides and cinéfilm, was a survey of the radiation and particle detectors used in modern nucleonic instruments. The paper included a discussion of the long-term stability of the detectors used in assay and other instruments, and summarized from this point of view the relative advantages and disadvantages of Geiger-Müller counters, proportional counters and scintillation counters. In particular, the paper gave consideration to the problem of industrial instrumentation where high reliability is the first consideration. Graphs were presented showing the changes of counting efficiency of typical Geiger-Müller counters over a period of time, and it was noted that with counters filled with argon and ethyl alcohol large changes in the efficiency can occur during their working life. Some 
of these changes are related to photoelectric effects of the counter, variations of temperature, electrostatic effects on the window of the counter, etc.; but it was pointed out that, even when proper precautions are taken to avoid variations in the measured counting-rate due to all these effects, some fluctuations in the counting-rate outside those to be expected on statistical grounds are still obtained.

Dr. Taylor showed some typical curves for a GeigerMüller counter and, in the cases shown, the countingrate dropped by about 5 per cent in the first few minutes of use and then fluctuated over narrower limits during the course of the next hundred hours or more, the fluctuations being much larger than would be expected from the statistics. He pointed out that in laboratory experiments it is always possible to wait until the counter has settled down, and it is usually possible to determine the counting-rate of the unknown and a standard source and express the first result in terms of the second. This course allows all but the short-term variations to be eliminated, and very accurate measurements can then be made. However, in many instruments this second measurement with a standard leads to considerable complication and, in the case of industrial instrumentation, it is desirable to use more stable detecting elements both on the grounds of reliability and on the grounds of time-saving. Dr. Taylor showed graphs which indicate that Geiger-Müller counters using bromine as the quenching agent give an improved performance from this point of view, but that still better results can be obtained by using a counter operating in the proportional region, and he showed an apparatus of this type capable of giving a precision of measurement of \pm 0.1 per cent and suitable for adaptation as an industrial instrument.

The two following papers were presented by $\mathrm{K}$. Fearnside and Dr. J. E. Johnston, respectively, and dealt with various aspects of the use of radioactive tracers in industry. Mr. Fearnside reviewed the development of the beta-ray thickness gauge and described a number of preferred designs, dealing not only with the transmission gauge, but also with the back-scattering type. He gave details of the applications of these gauges in industrial work and information about their reliability and accuracy. It appears that they are already being used on a large scale, but that the obvious step of controlling the manufacturing process by a servo-mechanism operating directly from the gauge still remains to be done. The gauges in use at the present time simply measure the thickness of the manufactured material (strictly speaking, the mass per unit area, which is usually proportional to the thickness), and an attendant must make the necessary alterations to the machine if the thickness is outside the prescribed limits.

Dr. Johnston's paper covered a very wide field of applications, and the discussion which followed was equally wide. The main applications discussed were : determination of the presence of arsenic impurity in the germanium used for making crystal diodes (Dr. E. Billig, of the Laboratory of the Associated Electrical Industries, Ltd.); determination of the rate of wear of the dies used for making wire (Dr. J. W. Drinkwater, of the Thornton Research Centre); testing the uniformity of mixed cattle foods (British Oil and Cake Mills); testing the ventilation system of a factory (British Belting and Asbestos, Ltd.) ; and measuring the rate of wear of blast furnace linings (Mr. E. W. Voice, of the British Iron and Steel Research Association).
One of the most interesting papers presented at the conference was on the subject of the self-balancing potentiometric recorder. This instrument has proved to be one of great versatility and importance, and R. S. Medlock and W. A. Kealy in their paper gave a comprehensive review of the applications of this type of instrument in research and in industrial measurements. A large proportion of the applications discussed were those which have been developed for use in atomic energy plants-a novel example being the measurement of pile power. It is interesting to note that the self-balancing potentiometric recorder competes in many cases with the more orthodox instruments depending on the use of thermionic valve circuits, and for certain classes of problem offers a simpler and more satisfactory solution; moreover, in many cases the completed instrument is more compact.

The remaining three papers dealt more particularly with design of nucleonic instruments and were as follows : "Counting of Random Pulses," by E. H. Cooke-Yarborough ; "Design of Portable Beta- and Gamma- Measuring Instruments," by E. Franklin and J. Hardwick; and "Pulse Circuits for the Millimicrosecond Range," by F. H. Wells. The paper on portable measuring instruments contained a great deal of new design data, and included details, to mention just a fow of the novel ideas presented, of the use of cold-cathode valves in place of filament valves in these instruments, the replacement of the battery by a clockwork-driven electric generator, the replacement of the meter presentation system by a 'fluid light' type of indicator, and the use of infinite-life Geiger counter tubes. The majority of the instruments described were designed for use in the nuclear physics laboratory, or for field use by geologists or mining engineers, but some of the designs would be suitable for use by the civil defence authorities in the event of atomic warfare.

The paper by Mr. Wells dealt with a subject of more general interest, and gave a review of the circuit techniques used in measurements on short pulses and time intervals between pulses in the range $10^{-9}-10^{-7}$ sec. The circuits described have been developed for use with scintillation and spark counters, and include pulse-shaping circuits, pulse generators, amplifiers, scaling circuits and oscilloscopes; and Mr. Wells gave examples of the use of these circuits for high-speed coincidence measurements, millimicrosecond time-interval measurements and fast counting. Probably the most interesting of the many circuits described is the fast coincidence circuit. This is essentially a mixer circuit, which will accept a pulse from each of two input channels and, if these pulses arrive at the same time, or within a given short time interval of each other, will give an output signal. In Mr. Wells's instrument the two input pulses are first shaped to give voltage impulses of defined duration, and these pulses are amplified so that each output pulse is of $10 \mathrm{~V}$. amplitude. If, therefore, pulses from the two input channels arrive at exactly the same time, a double-amplitude pulse of $20 \mathrm{~V}$. will be obtained. Hence, coincident pulses may be sorted out from non-coincident pulses by the use of a pulsesmplitude discriminator set to $15 \mathrm{~V}$. following the amplifier, the output of the amplifier being the coincidence unit output-pulse. Mr. Wells gave details of a number of circuits he has built making use of this principle, and reported that the minimum coincidence resolving time readily obtainable is 10 millimicroseconds.

DENIS TAYLOR 